\title{
HABITAT HETEROGENEITY AND BUTTERFLY DIVERSITY OF KULDIHA WILDLIFE SANCTUARY, ODISHA, INDIA
}

\author{
Paria, S., S. Ghosh ${ }^{1}$, P. C. Mardaraj ${ }^{2}$, P. H. Mallick and S. K. Chakraborty \\ Department of Zoology, Vidyasagar University, Midnapore-721102, West Bengal, India; ${ }^{1}$ Post \\ Graduate Department of Zoology, Govt. Bethune College, 181, Bidhan Sarani, Kolkata, West Bengal, \\ India; ${ }^{2}$ Department of Forest, Odisha, Wildlife Biologist, IUCN/SSC BSG, At-Palace, Nilgiri, Post- \\ Rajnilgiri, Balasore, Odisha-756040, India
}

\begin{abstract}
The present communication aims to highlight the trend of distribution of butterfly species in and around the protected forest areas of Kuldiha Wildlife Sanctuary (KWS), characterized with habitat heterogeneity with respect to vegetational assemblages, soil profiles and water bodies in comparison to butterfly diversity in other parts of India and neighbouring country. Butterfly diversity and distribution along with the seasonal gradients across three landscape areas, viz., LSE1, LSE2 and LSE3 were recorded at Kuldiha, Odisha, India using line transect. The method has generated baseline information relating not only to diversity, but also synchronization with vegetative growth coupled with phenological periodicity, and habitat heterogeneity of this aesthetically celebrated faunal component in a tropical deciduous evergreen forest landscape. A total of 95 species belonging to 63 genera and 5 families was encountered throughout the whole study period (Winter, 2015 to Summer, 2018) excepting the monsoon months. Post monsoon lepidopteran assemblage pattern was quite different from that of the pre-monsoon. Maximum diversity was observed in the LSE2, i.e. Mixed forest type exposed to certain levels of anthropogenic interactions. Present studies are considerably contribute to the ecobiology of the studied flagship species in particular and as a step towards holistic conservation strategy of an ecopotential sanctuary in the tropical country, India in general.
\end{abstract}

Key words: Bioindicator, butterfly diversity, conservation, Kuldiha Wildlife Sanctuary.

\section{INTRODUCTION}

India, being a megadiversity region, is gifted with diversified form of bio-eco-geographical zones in tune with the regional, ecological, climatological set-up. The present research site, Kuldiha Wildlife Sanctuary (KWS) is a well-protected and stable ecozone. It satisfies the characteristics of tropical deciduous forest system harbouring a rich diversity of wild fauna which includes butterflies; being a flagship species butterflies cater the need of ecosystem functioning by their roles as pollinators and an effective component of food chain-food web system. Butterflies are very sensitive biodiversity components. They are very vitally used to assess the ecological changes (Koh 2007). Significance of butterflies as bioindicator against climatic changes was highlighted by Bashar (2010). For implementing potential conservational strategies mapping and monitoring of biological diversity is of utmost importance. The importance of the pivotal role is played by the butterflies as focal species in biodiversity monitoring studies (Kunte 1999). So far, 1,318 species of butterflies (Varshaney and Smetacek 2015) have been recorded from different eco-zones of India. One hundred forty-five species of butterflies were recorded from the eight study sites, of which 62 species were new records for the Nagpur city (Tiple and Khurad 2009). Seventy one (71) species of butterflies were found in Anuka reserve forest, Jharkhand (Singh 2010). Fifty nine (59) species of 48 genera belonging to five families were recorded in Trishna Wildlife Sanctuary, Tripura, northeast India (Majumder et al. 2012). Two hundred and forty-three species of butterflies were recorded from Jones Estate, Uttarakhand, India (Smetacek 2012). Twenty-nine lepidopteran insect species belonging to 26 genera of 10 families have been recorded from eight different sites in the coastal areas of Midnapore (East), West Bengal, India (Jana et al. 2013). A total of 49 species of butterflies under 36 genera of 5 families was recorded from 
May 2013 to April 2014 in the Sarojini Naidu College campus, Dum Dum, Kolkata (Nair et al. 2014). In Sunabeda Wildlife Sanctuary in Nuapada district of Odisha, a total number of 101 species of butterflies belonging to five families was documented through a five months' survey (Palei and Rath 2014). A total of 136 species of butterflies belonging to 87 genera representing 5 families was found at two different study sites in the fringe area of Similipal Biosphere Reserve during January 2014 to November 2015 (Payra et al. 2016).

The present study has attempted to highlight in recording diversity, habitat preference and seasonality of butterflies. This study is also preparing their relative abundance profile in a heterogeneous tropical deciduous forest system, in KWS at the scale of three different landscape elements of eastern India.

\section{Study area}

\section{MATERIAL AND METHODS}

The Kuldiha Wildlife Sanctuary (KWS) covers an area of 272.75 sq. kms, situated at Nilgiri Civil Sub division in Balasore (Wildlife) division, between $21^{\circ} 20^{\prime}$ to $21^{\circ} 30^{\prime} \mathrm{N}$ and $86^{\circ} 30^{\prime}$ to $86^{\circ} 45^{\prime} \mathrm{E}$, merging with Similipal Forest (Fig. 1). There were three landscape elements: LSE1-Closed/dense canopy, LSE2Mixed forest, and LSE3-interspersed water bodies.

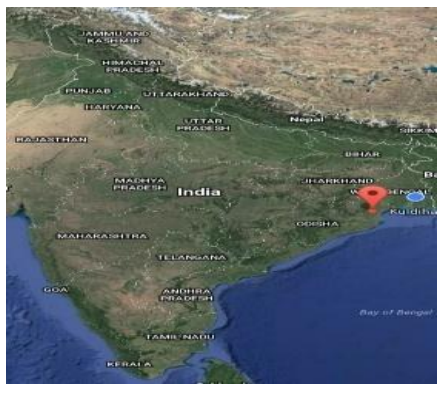

a

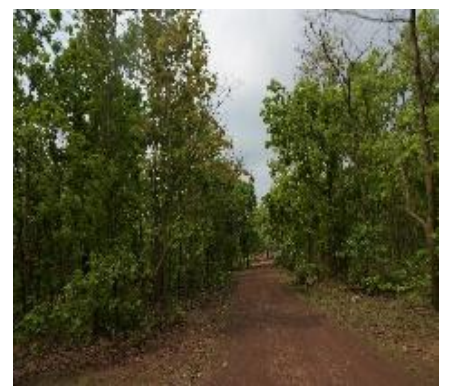

b

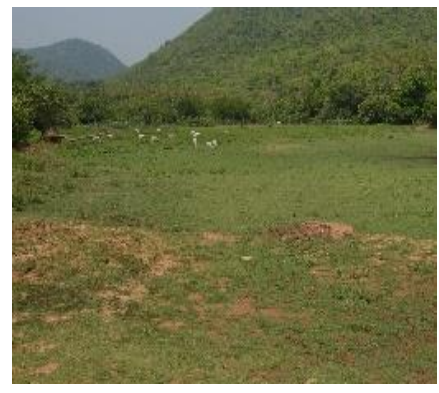

c

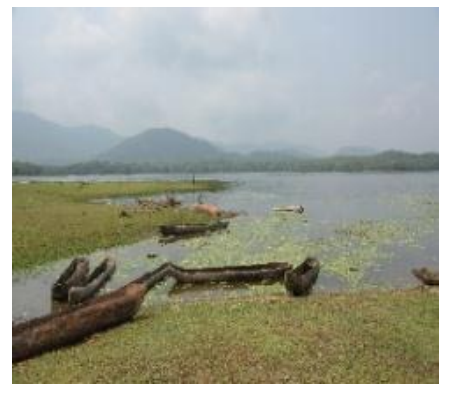

d

Fig. 1. Study sites: a. map indicating Kuldiha Wildlife Sanctuary (KWS), b. Landscape Element 1 (LSE1-Closed / Dense canopy), c. Landscape Element 2 (LSE2-Mixed forest) and d. Landscape Element 3 (LSE3-Interspersed Water bodies).

\section{Climatic condition}

Based on rainfall and temperature three seasons viz. Pre-monsoon (March to June), monsoon (July to October) and post monsoon (November to February) are existed. The temperature ranges from $5^{\circ}-42^{\circ} \mathrm{C}$. The relative humidity has been recorded from $62 \%$ to $88 \%$. The annual average precipitation hovers around $1,460 \mathrm{~mm}$.

\section{Duration of Study and Sampling process}

The study was conducted as a part of pilot survey to collect, preserve and photograph different butterfly taxa from Kuldiha sanctuary. This investigation gives special emphasis on seasonality and relative abundance from selected study sites. Species level identification was done following Kehimkar (2008) and Varshney and Smetacek (2015).

The observation was set on the basis of the probability of sighting. Seasonal availability was determined by presence-absence scoring method, and percentage calculation. Pollard transect walk method with few modifications was adopted for recording the field data (Pollard 1977, Pollard and Yates1993). 


\section{RESULTS AND DISCUSSION}

Ninety five butterfly species (95) was recorded from the present study. They exhibiting differential mode of habitat preference and also seasonality in the studied tropical bio-geographical regions of Eastern India (Table 1, Fig. 1). The present study revealed that the LSE2 harboured the maximum (39\%) representation of butterfly species followed by LSE1 (32\%) and LSE3 (29\%) (Fig. 2). Some ericaceous species have been found to occur in various habitats within forest patches (Haider 2017, Lodh and Agarwala 2015). The significant canopy coverage alongside vegetation patches composed of climbers, herbs, shrubs, trees representing mixed forest during all seasons. The species are Pelopidas conjuncta, Spialia galba, Borbo cinnara (Hesperids), Graphium sarpedon, Papilio polymnestor, Atrophaneura aristolochiae, Atrophaneura hector (Papilionids), Eurema hecabe, Eurema brigitta, Appias lyncida (Pierids), Castalius rosimon, Deudorix isocrates, Neopithecops zalmora, Chilades lajus (Lycaenids), and Junonia almana, Ypthima baldus, Hypolimnas misippus (Nymphalids). These butterflies were also reported to frequently visit water bodies interspersed with shrubs/herbs and macrophytes. Those are generally of polyphagous and with broader range of host plant preferences. Interestingly, few specialized sensitive species viz. Eurema andersonii (Pieridae), Arhopala amantes, Anthene lycaenina (Lycaenids), Charaxes marmax, Junonia atlites, Danaus genutia (Nymphalidae) were found only at LSE1, whereas Leptosia nina, Catopsilia pomona (Pieridae), Tarucus nara, Euchrysops chejus, Freyeria trochylus, Curetis acuta (Lycinidae), Mycalesis perseus, Mycalesis mineus, Ypthima huebneri, Junonia almanac (Nymphalidae) utilizing only specific floral members of the forests. These butterflies also showed selective preferences towards host plants choice. Those species are with restricted microhabitat occupancy for performing their crucial life history strategies viz. courtship, oviposition, larval development, mud-puddling (Leps and Spitzer 1990). The alternative (except nectar) source of organic resource utilization pattern found to be restricted only to LSE2.

Table 1. List of butterfly species found in LSE1 (canopy forest), LSE2 (mixed forest) and LSE3 (interspersed water bodies) in KWS (Kuldiha Wildlife Sanctuary) (Kehimkar 2008).

\begin{tabular}{|c|c|c|c|c|c|c|}
\hline \multirow[t]{2}{*}{ Family } & \multirow[t]{2}{*}{ Scientific name } & \multirow[t]{2}{*}{ Common name } & \multicolumn{3}{|c|}{ Distribution status } & \multirow{2}{*}{$\begin{array}{l}\text { Relative } \\
\text { abundance }\end{array}$} \\
\hline & & & LSE1 & LSE2 & LSE3 & \\
\hline \multirow[t]{9}{*}{ Hesperiidae } & Parnara guttatus $\mathbf{P}-\mathbf{A}-\mathbf{v}$ & Straight Swift & + & + & + & 0.90 \\
\hline & Spialia galba $\mathbf{P}-\mathbf{A}-\mathbf{i i}$ & Indian Skipper & + & + & + & 1.32 \\
\hline & Pseudocoladenia dan $\mathbf{P}-\mathbf{A}-\mathbf{i v}$ & Fulvous Pied Flat & + & + & + & 1.00 \\
\hline & Borbo cinnara $\mathbf{P}-\mathbf{A}$-iii & Rice Swift & + & + & + & 2.24 \\
\hline & Pelopidas conjuncta $\mathbf{P}-\mathbf{A}-\mathbf{i}$ & Conjoined Swift & + & + & + & 0.71 \\
\hline & Pelopidas mathias $\mathbf{P - B - i}$ & Small Branded swift & + & + & + & 0.84 \\
\hline & Udaspes folus P-B-ii & Grass Demon & + & + & + & 0.85 \\
\hline & Telicota colon P-B-iii & Pale Palm swift & - & + & + & 0.91 \\
\hline & Borbo bevani P-B-iv & Lesser Rice Swift & - & + & - & 0.94 \\
\hline \multirow[t]{10}{*}{ Papilionidae } & Graphium Agamemnon P-D-iii & Tailed jay & + & + & + & 0.37 \\
\hline & Lamproptera meges & Green Dragon tail & - & + & + & 0.13 \\
\hline & Graphium sarpedon & Common Bluebottle & + & + & + & 2.01 \\
\hline & Graphium nomius P-D-ii & Spot Sword Tail & + & + & + & 0.34 \\
\hline & Graphium doson P-D-i & Common Jay & + & + & + & 0.79 \\
\hline & Chilasa clytia P-C-iv & Common Mime & + & + & + & 1.19 \\
\hline & Papilio demoleus P-C-v & Lime Swallow Tail & + & + & - & 0.29 \\
\hline & Papilio polytes $\mathbf{P}-\mathbf{C}$-iii & Common Mormon & + & + & + & 4.25 \\
\hline & Papilio nephelus & Yellow Helen & + & + & + & 0.40 \\
\hline & Papilio polymnestor $\mathbf{P}-\mathbf{C}-\mathbf{i}$ & Blue Mormon & + & + & + & 0.63 \\
\hline
\end{tabular}




\begin{tabular}{|c|c|c|c|c|c|c|}
\hline & Papilio paris & Paris Peacock & + & + & + & 0.84 \\
\hline & Papilio crino $\mathbf{P}-\mathbf{B}-\mathbf{v}$ & Common Banded Peacock & + & + & - & 0.40 \\
\hline & Atrophaneura aristolochiae $\mathbf{P}-\mathbf{C}-\mathbf{i i}$ & Common Rose & + & + & + & 3.61 \\
\hline & Atrophaneura hector & Crimson Rose & + & + & + & 2.77 \\
\hline \multirow[t]{14}{*}{ Pieridae } & Eurema andersonii P-E-i & One spot Small Grass Yellow & + & - & - & 0.34 \\
\hline & Eurema hecabe P-D-v & Common Grass Yellow & + & + & + & 3.30 \\
\hline & Eurema blanda & Three spot Grass Yellow & + & + & + & 1.61 \\
\hline & Eurema brigitta P-D-iv & Small Grass Yellow & + & + & + & 1.11 \\
\hline & Delias eucharis $\mathbf{P}-\mathbf{E}-\mathbf{i i}$ & Common Jezebel & + & + & + & 4.51 \\
\hline & Belenois aurota $\mathbf{P}$-E-iv & Pioneer & - & - & + & 0.11 \\
\hline & Leptosia nina $\mathbf{P}-\mathbf{F}-\mathbf{i i}$ & Psyche & - & + & - & 2.16 \\
\hline & Cepora nerissa $\mathbf{P}-\mathbf{F}-\mathbf{i}$ & Common Gull & + & + & - & 0.79 \\
\hline & Pareronia valeria $\mathbf{P}-\mathbf{E}-\mathrm{iii}$ & Common Wanderer & - & + & + & 1.03 \\
\hline & Catopsilia pyranthe $\mathbf{P}-\mathbf{F}$-iii & Mottled Emigrant & + & + & - & 0.21 \\
\hline & Catopsilia Pomona $\mathbf{P}-\mathbf{F}-\mathbf{i v}$ & Common Emigrant & - & + & - & 0.11 \\
\hline & Appias lyncida & Chocolate Albatross & + & + & + & 0.90 \\
\hline & Appias albino & Common Albatross & + & + & - & 0.66 \\
\hline & Ixias pyrene $\mathbf{P}-\mathbf{E}-\mathbf{v}$ & Yellow Orange Tip & + & - & - & 0.67 \\
\hline \multirow[t]{24}{*}{ Lycaenidae } & Caleta caleta $\mathbf{P}-\mathbf{H}-\mathbf{i i}$ & Angled Pierrot & + & + & - & 1.08 \\
\hline & Castalius rosimon P-G-iii & Common Pierrot & + & + & + & 2.66 \\
\hline & Taraka hamada & Forest Pierrot & + & + & - & 0.61 \\
\hline & Tarucus nara $\mathbf{P}-\mathbf{G}-\mathbf{i}$ & Rounded Pierrot & - & + & - & 0.42 \\
\hline & Tarucus indica & Pointed Pierrot & - & + & + & 1.06 \\
\hline & Leptotes plinius $\mathbf{P}-\mathbf{H}-\mathbf{i i i}$ & Zebra blue & + & + & + & 2.61 \\
\hline & Spindasis vulcanus $\mathbf{P}-\mathbf{G}-\mathbf{i i}$ & Common Silverline & - & + & + & 1.03 \\
\hline & Spindasis lohita & Long Banded Silverline & + & + & + & 1.93 \\
\hline & Lampides boeticus $\mathbf{P}-\mathbf{H}-\mathbf{i v}$ & Pea Blue & - & + & + & 0.40 \\
\hline & Acytolepis puspa $\mathbf{P}-\mathbf{G}-\mathbf{i v}$ & Common Hedge Blue & + & + & + & 1.48 \\
\hline & Euchrysops cnejus $\mathbf{P}-\mathbf{F}-\mathbf{v}$ & Gram Blue & - & + & - & 1.42 \\
\hline & Deudorix isocrates & Guava Blue & + & + & + & 0.69 \\
\hline & Arhopala amantes & Large Oak-Blue & + & - & - & 0.66 \\
\hline & Neopithecops zalmora & Quaker & + & + & + & 3.75 \\
\hline & Heliophorus epicles $\mathbf{P}-\mathbf{G}-\mathbf{v}$ & Purplr Sapphire & + & + & - & 0.79 \\
\hline & Freyeriatrochylus & Grass Jewel & - & + & - & 1.11 \\
\hline & Chilades lajus & Lime Blue & + & + & + & 3.40 \\
\hline & Curetis bulis & Bright Sunbeam & - & + & + & 0.42 \\
\hline & Curetis acuta & Angled Sunbeam & - & + & - & 0.08 \\
\hline & Anthene emolus & Common Ciliate Blue & - & + & + & 0.95 \\
\hline & Anthene lycaenina & Pointed Ciliate Blue & + & - & - & 0.16 \\
\hline & Zizina otis $\mathbf{P - H - v}$ & Lesser Grass Blue & + & + & + & 1.48 \\
\hline & Zizeeria karsandra $\mathbf{P}-\mathbf{H}-\mathbf{i}$ & Dark Grass Blue & - & + & + & 1.77 \\
\hline & Zizula hylax & Tiny Grass Blue & + & + & + & 2.85 \\
\hline \multirow[t]{5}{*}{ Nymphalidae } & Acraea violae P-L-iv & Tawny Coster & - & - & + & 0.95 \\
\hline & Ariadne ariadne $\mathbf{P}-\mathbf{N}$-iii & Angled Castor & - & - & + & 0.78 \\
\hline & Euploea core P-I-i & Common Crow & - & + & - & 0.95 \\
\hline & Tirumala limniace $\mathbf{P}-\mathbf{J}-\mathbf{i}$ & Blue Tiger & - & + & - & 1.48 \\
\hline & Euthalia nais P-M-ii & Baronet & - & - & + & 0.32 \\
\hline
\end{tabular}




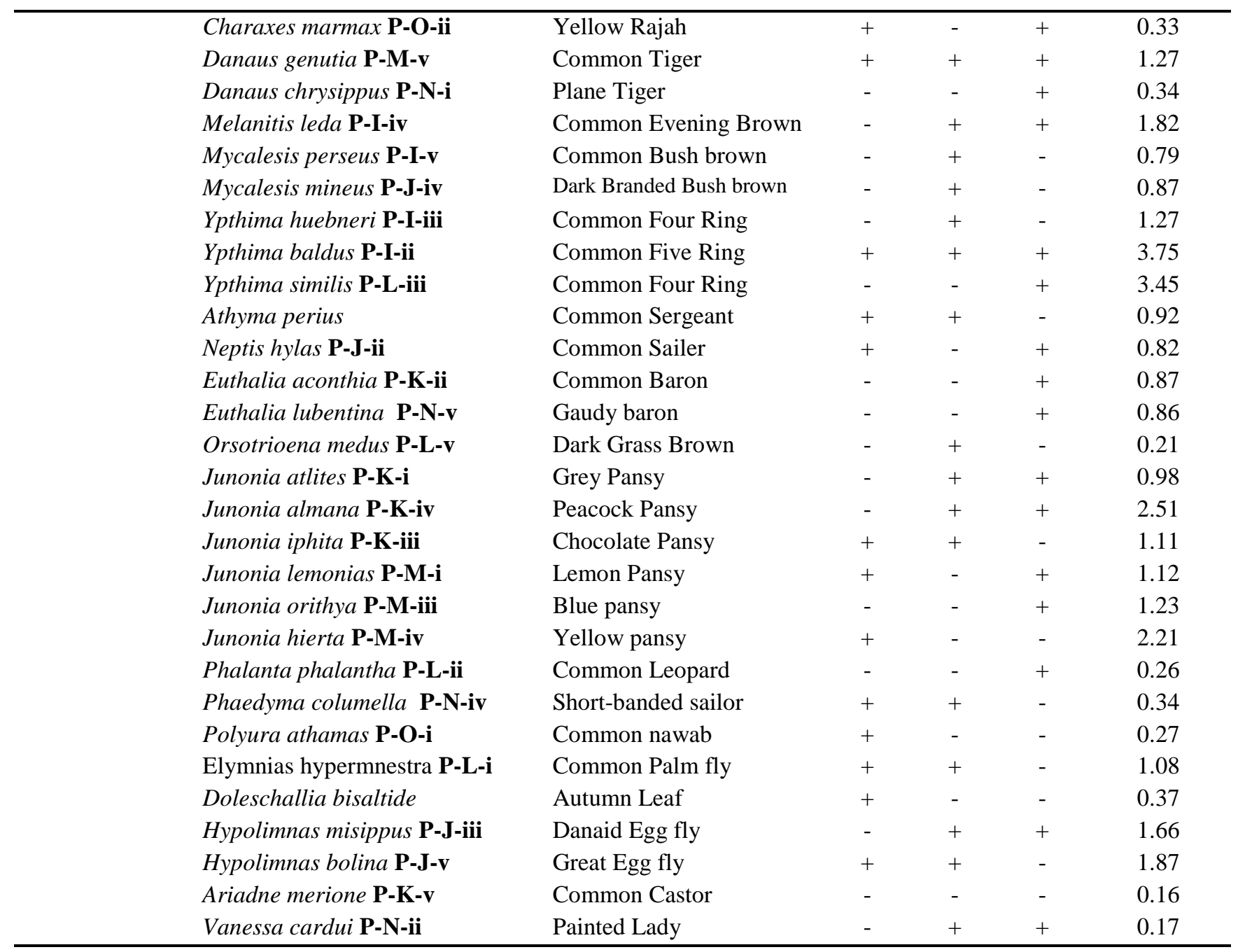

Exclusive visitors to LSE3 were reported to be species like Belenois aurota (Pieridae), Euthalia aconthia and Danaus chrysippus (Nymphalidae). On the basis of overall abundance pattern, Curetis acuta (0.08), Belenois aurota and Catopsilia pomona (0.11), Lamproptera meges (0.13) were estimated significant lower relative abundance whereas, Delias eucharis (4.51), Papilio polytes (4.25) were with considerably higher relative abundance (Table 1). Considering the observed documents, it has been found that hesperids ( 7 species) were prevailed almost equally through different LSEs. Highest number of species belonging to Papilionidae (14species) were recorded at LSE2, as that of Pieridae (11 species), Lycaenidae (22 species) and Nymphalidae (16 species). Lowest number of species from Papilionidae (12 species) were observed to visit LSE3 as that of Pieridae (7 species) and Nymphalidae (12 species). LSE1 has been found to harbor least number of lycaenids (14 species) (Fig. 3). During pre-monsoon, nymphalids were preferred LSE1 (13 species) and LSE2 (16 species), respectively. On the other hand LSE3 was found to be most preferred by lycaenids (16 species). LSE1 was considered to be least preferred by hesperids (3 species), similarly in LSE2 (6 species of hesperids). LSE3 was least visited by the members of Papilionidae and Pieridae ( 3 species from each). In post monsoon, it was found that papilionids were availed mostly in LSE1 (9 species) and LSE2 (12 species), respectively. 


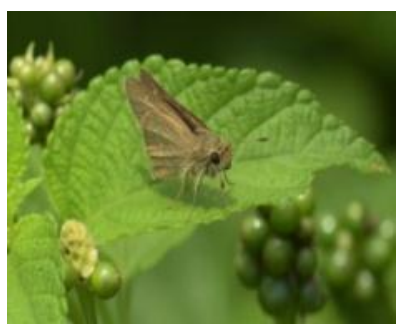

P-A-i

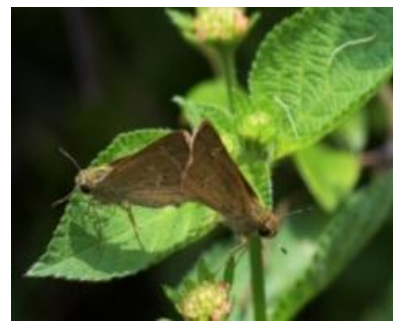

P-A- V

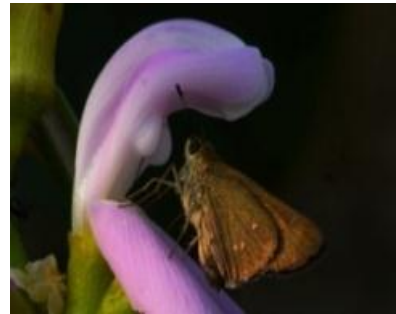

P-B -iv

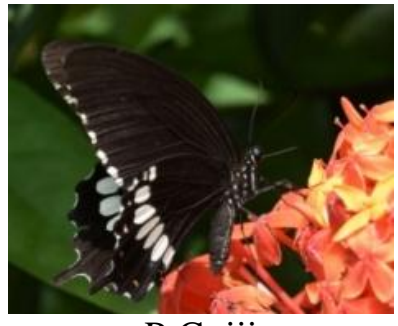

P-C -iii

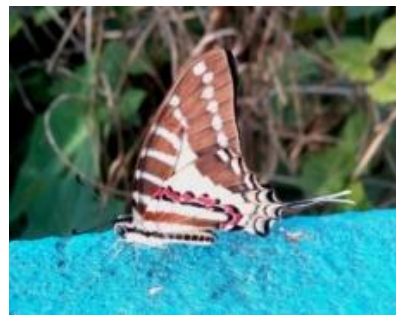

P-D-ii

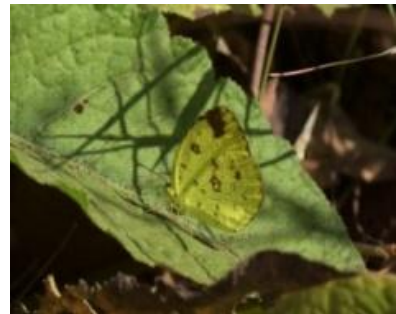

P-E-i

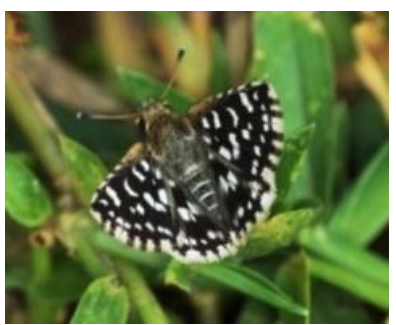

P-A-ii

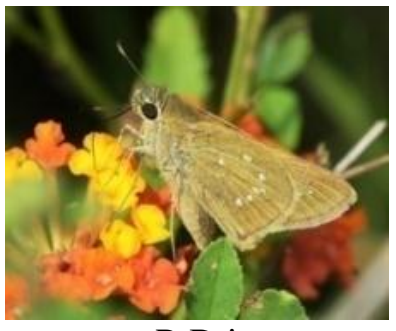

P-B-i

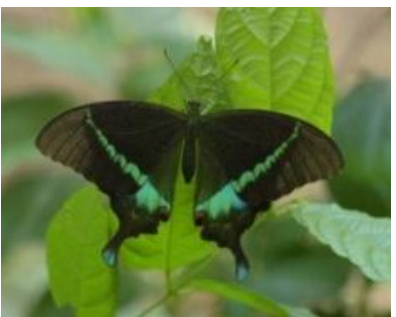

P-B- V
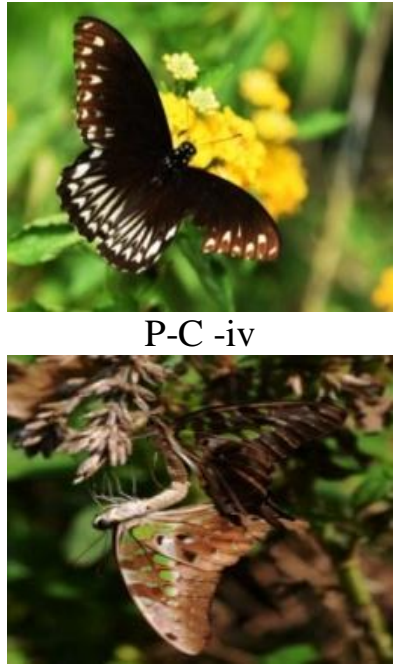

P-D-iii

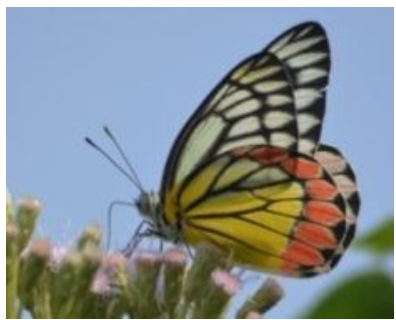

P-E-ii

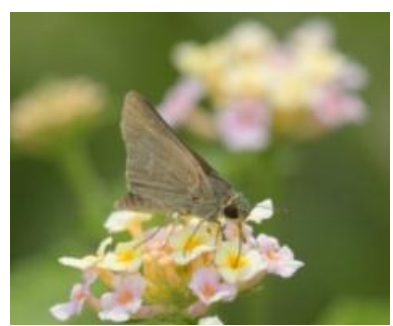

P-A-iii

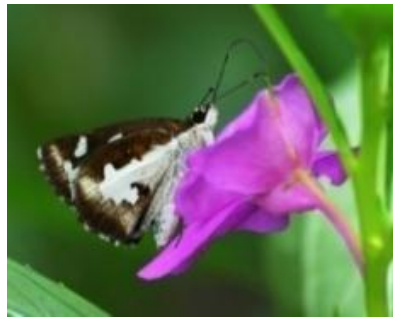

P-B -ii

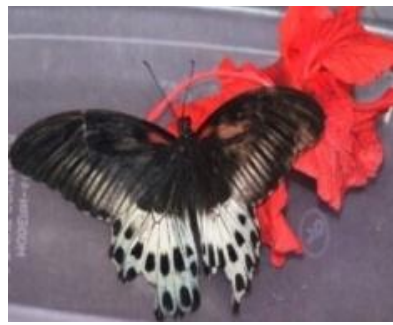

P-C-i

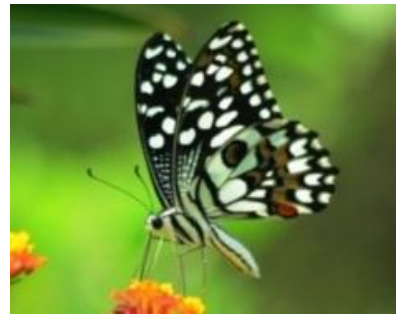

P-C -V

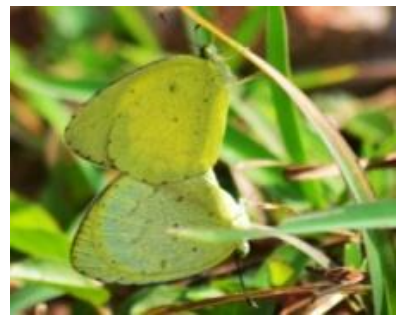

P-D-iv

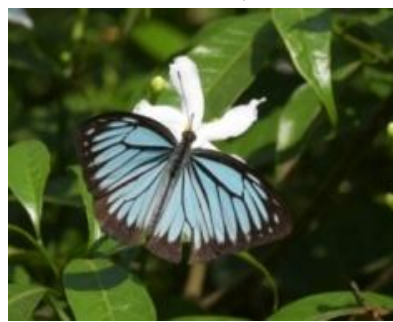

P-E-iii

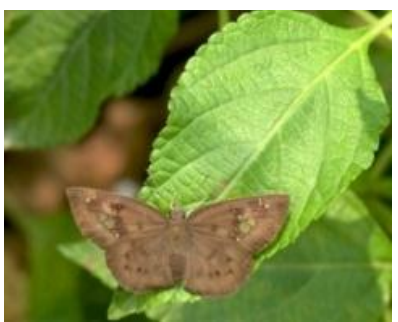

P-A-iv

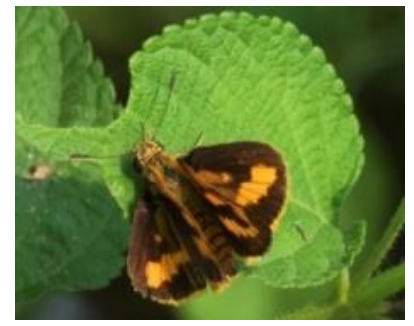

P-B -iii

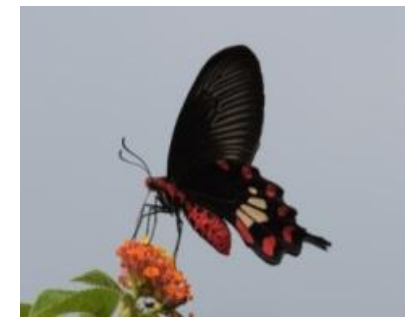

P-C -ii

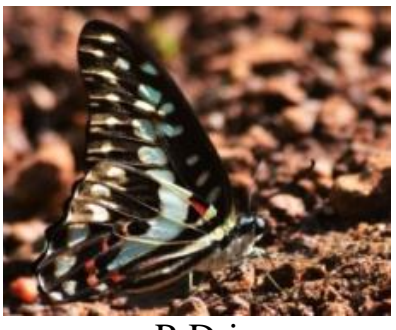

P-D-i

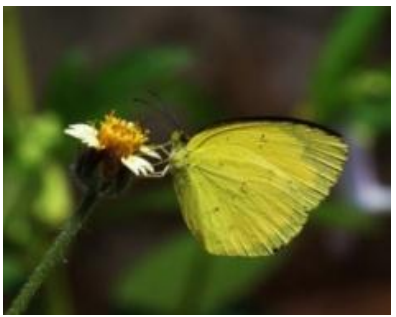

P-D-V

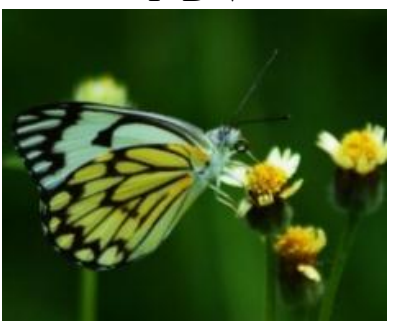

P-E-iv

Fig. 1a. Pictorial representation of major butterfly species. 


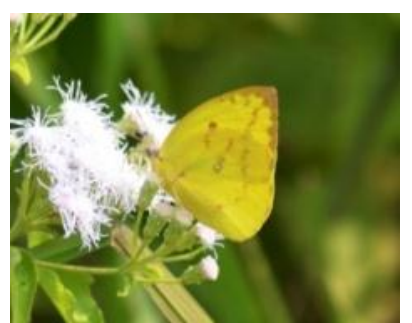

P-E-V

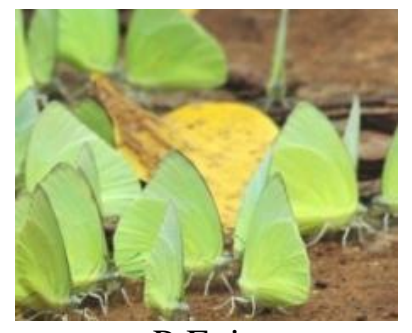

$\mathrm{P}-\mathrm{F}$-iv

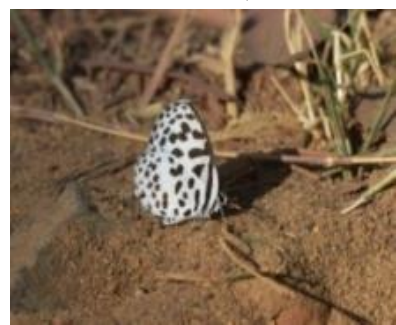

P-G-iii

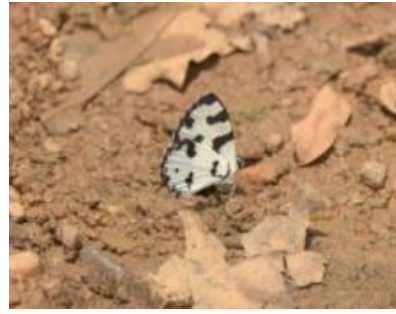

$\mathrm{P}-\mathrm{H}-\mathrm{ii}$

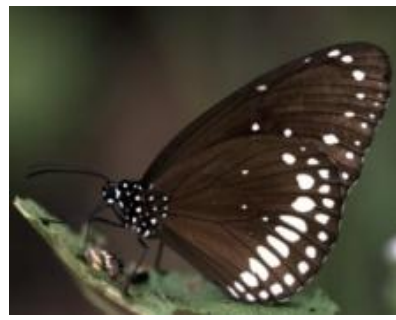

P-I-i

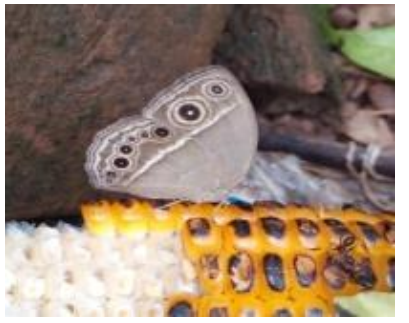

P-I-v

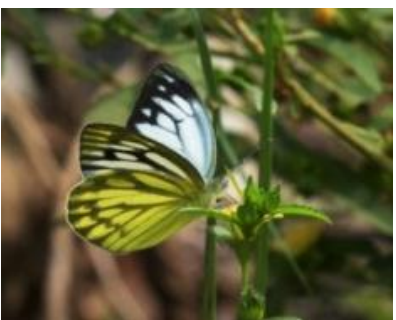

P-F-i

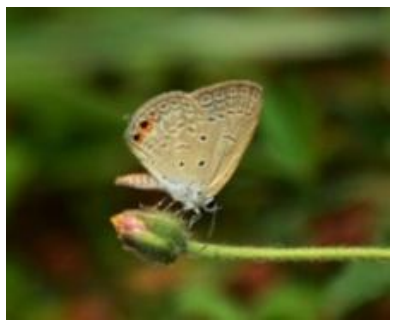

P-F -V

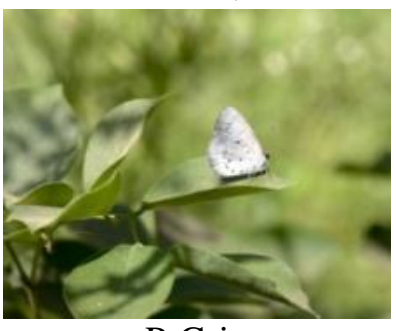

P-G-iv

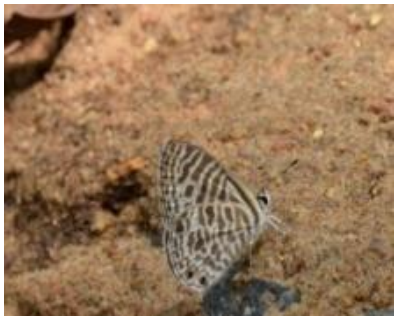

P-H-iii

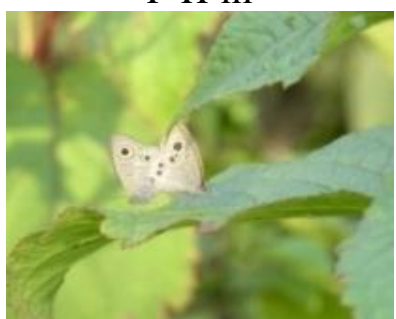

P-I-ii

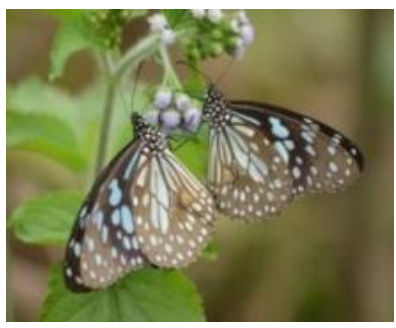

P-J-i

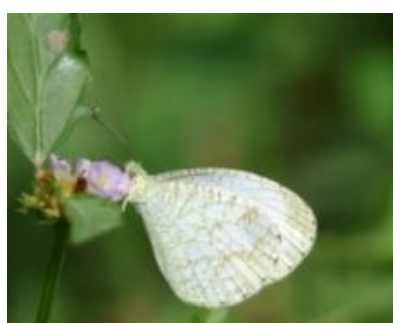

P-F-ii

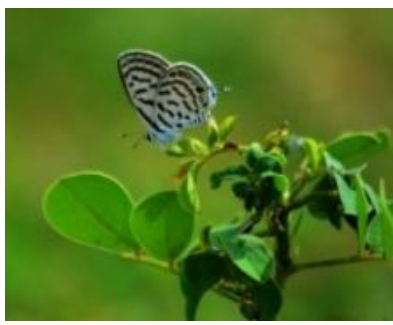

P-G-i

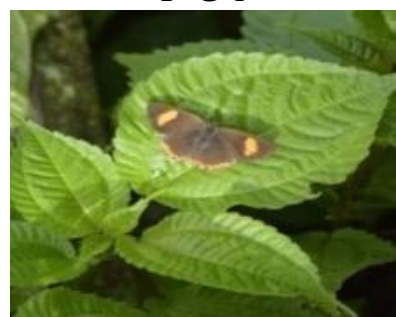

P-G-v

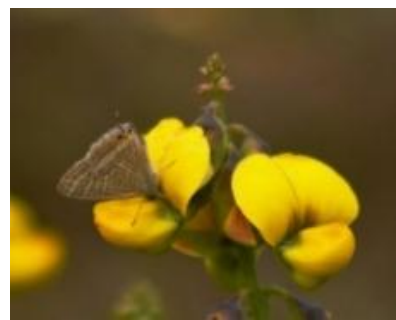

P-H-iv

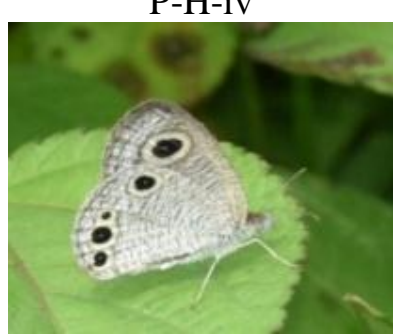

P-I-iii

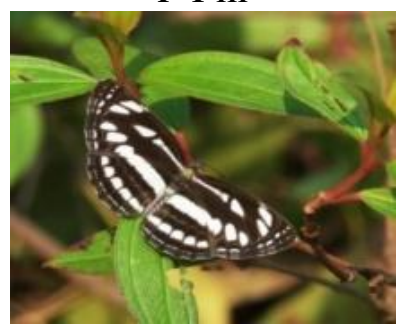

P-J-ii

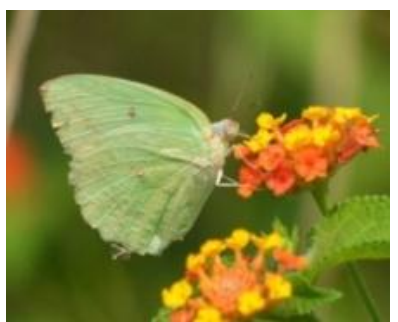

P-F-iii

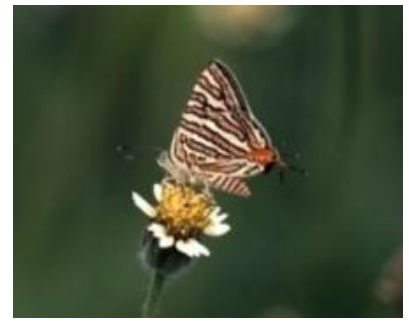

P-G-ii

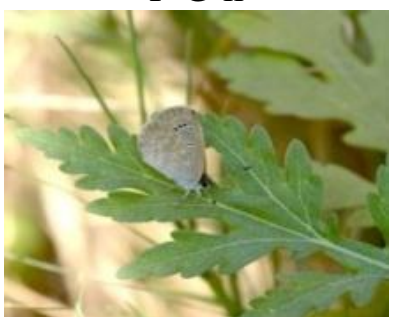

P-H-i

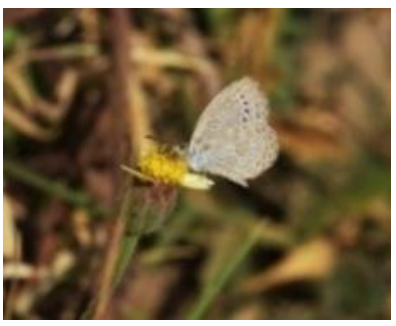

P-H-v

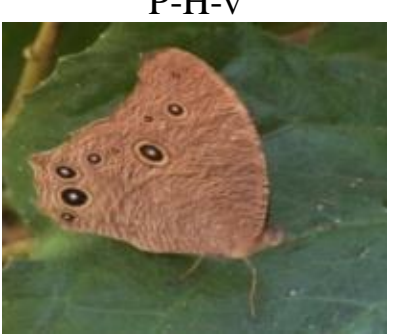

P-I-iv

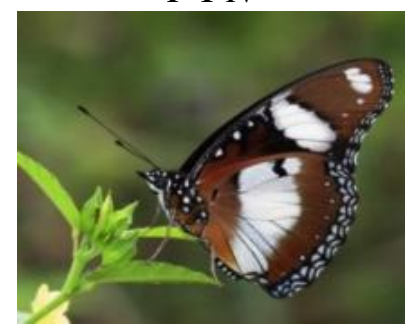

P-J-iii

Fig. 1b. Pictorial representation of major butterfly species. 


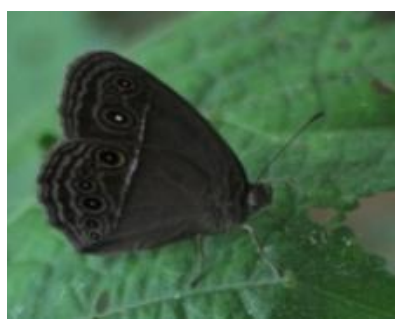

P-J-iv
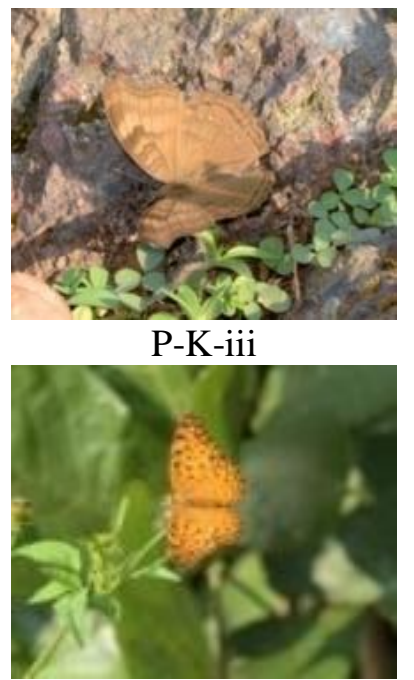

P-L-ii

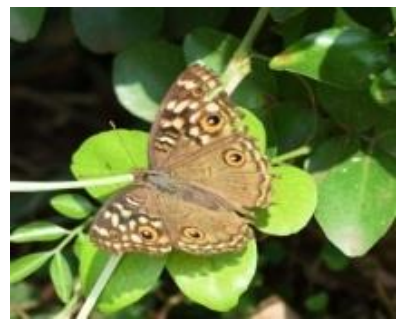

P-M-i

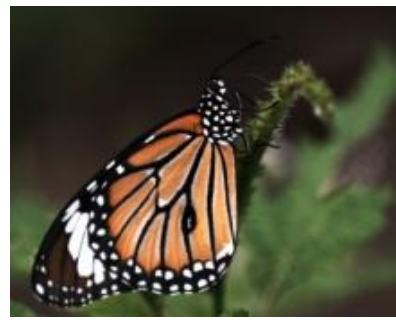

P-M-v

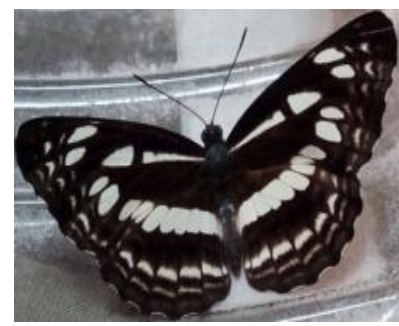

$\mathrm{P}-\mathrm{N}-\mathrm{iv}$

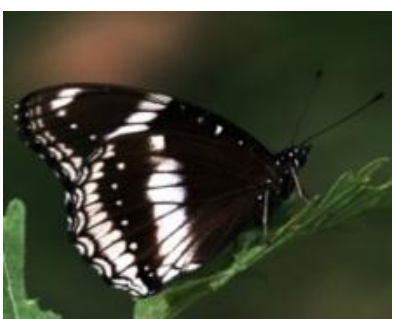

P-J-V

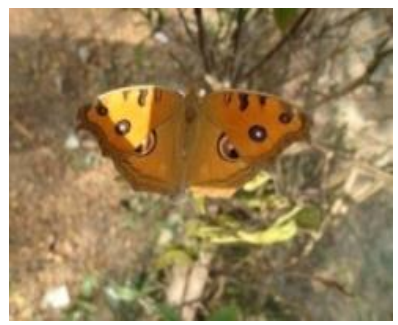

P-K-iv

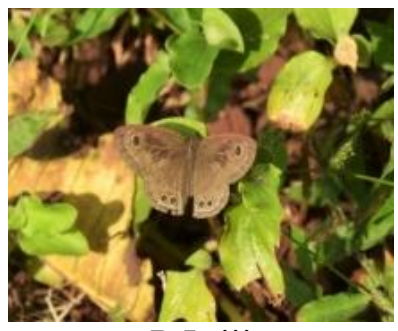

P-L-iii

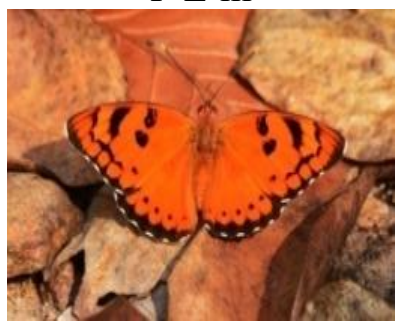

P-M-ii

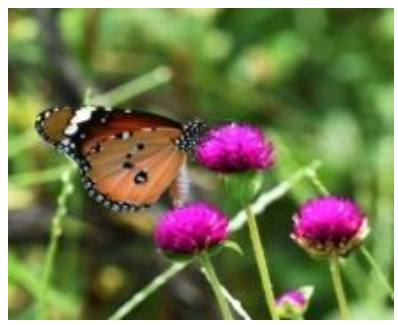

$\mathrm{P}-\mathrm{N}-\mathrm{i}$

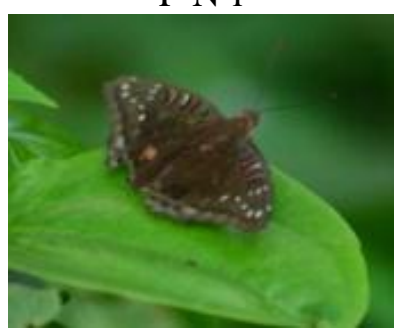

$\mathrm{P}-\mathrm{N}-\mathrm{v}$

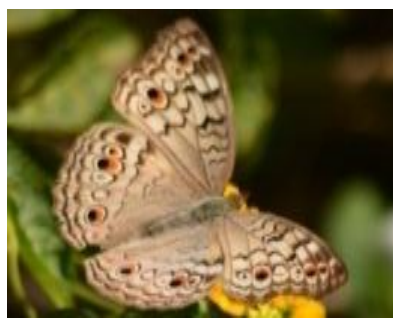

$\mathrm{P}-\mathrm{K}-\mathrm{i}$

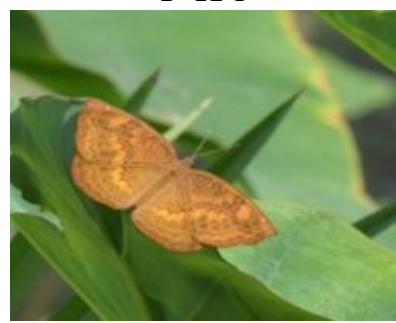

$\mathrm{P}-\mathrm{K}-\mathrm{v}$

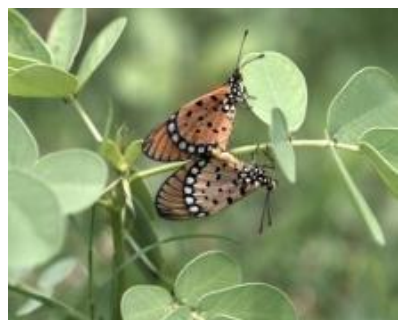

P-L-iv

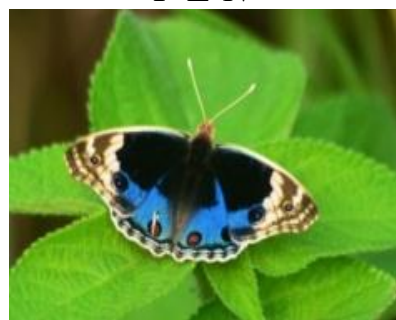

P-M-iii

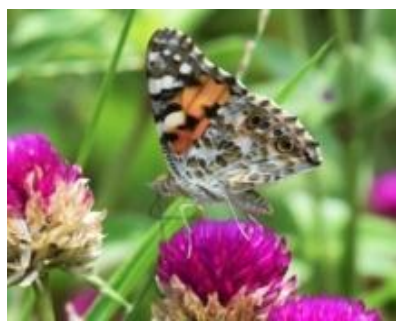

$\mathrm{P}-\mathrm{N}-\mathrm{ii}$

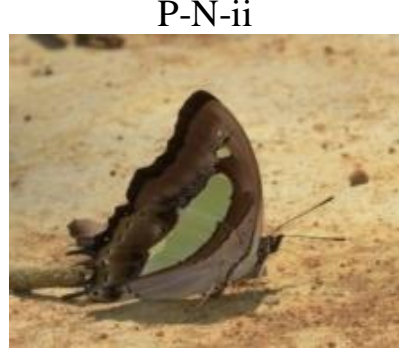

$\mathrm{P}-\mathrm{O}-\mathrm{i}$

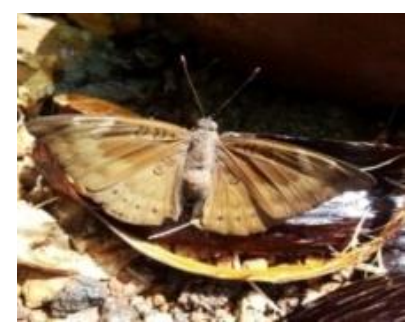

P-K-ii

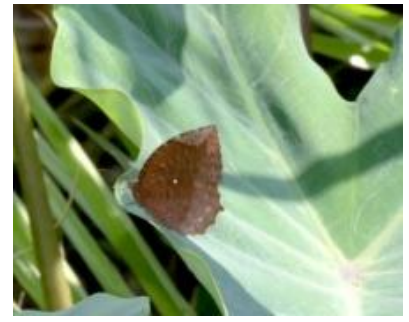

P-L-i

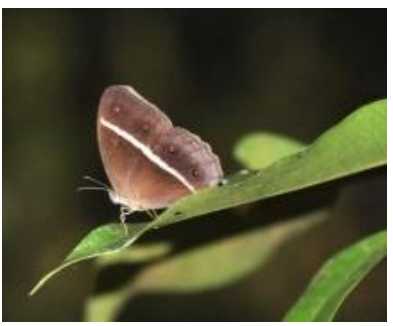

P-L-V

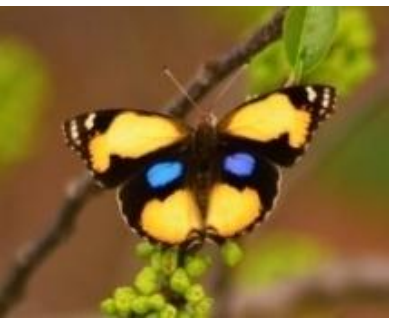

P-M-iv

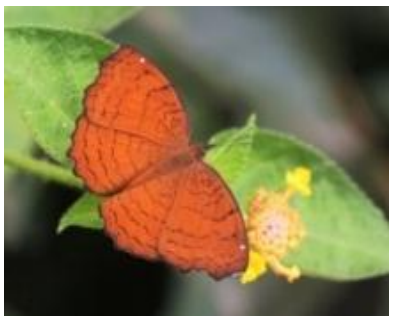

$\mathrm{P}-\mathrm{N}-\mathrm{iii}$

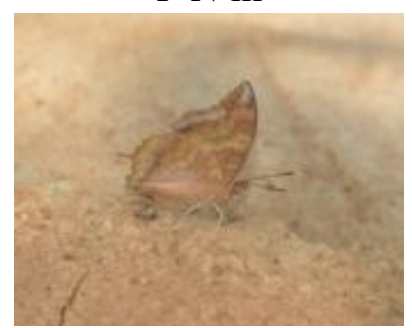

P-O-ii

Fig. 1c. Pictorial representation of major butterfly species. 
Considerably less number of hesperids and pierids ( 3 species from each family) was found to be present in LSE1. Hesperid butterflies were also least prevalent in LSE2 (6 species) and LSE3 (4 species). At LSE3, butterfly species under lycaenids were found in highest numbers (15 species). A thorough study more or less on similar distributed pattern was conducted in the 36 forest areas in Bangladesh for the duration of ten years (Bashar 2015). The authors worked on the butterflies of ten different families of Bangladesh. Their results more or less are harmonious to the present study results (Bashar 2014).

Habitat quality, edge effects and landscape matrix attributes were represented as factors leading to differential occurrence of species. The temporary habitat shrinkage with the partial drying up of mixed forest patches and interspersed water bodies was avail in pre-monsoon. In the present study, mixed forest habitat considered as transitional habitat or 'edge' between canopy forest and interspersed water bodies. Highest number of species occurrence (39\%) in this habitat caused by three hypothetical mechanisms. The mechanisms are, firstly excess of individuals' dispersal from adjacent habitats leading to an accumulation of species of both habitats near the 'edge'; secondly, driven by the presence of essential resources at the 'edge', which may be rare or absent in adjacent patches and finally by the increased probability to access to the complementary resources located in adjacent patches (Figs. 1, 2, 3 and 4). The most significant factors (nutritional and other resources, and the interactions among different species) are determining the individual species' ability to utilize the habitats optimally (Ries and Sisk 2004, Bashar 2014, Aich et al. 2016, Alam 2017).

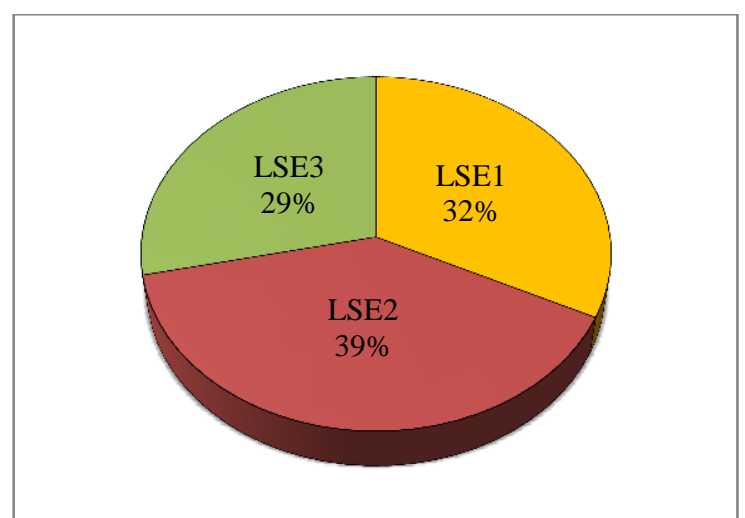

Fig. 2. Occurrence of butterfly species at different LSEs.

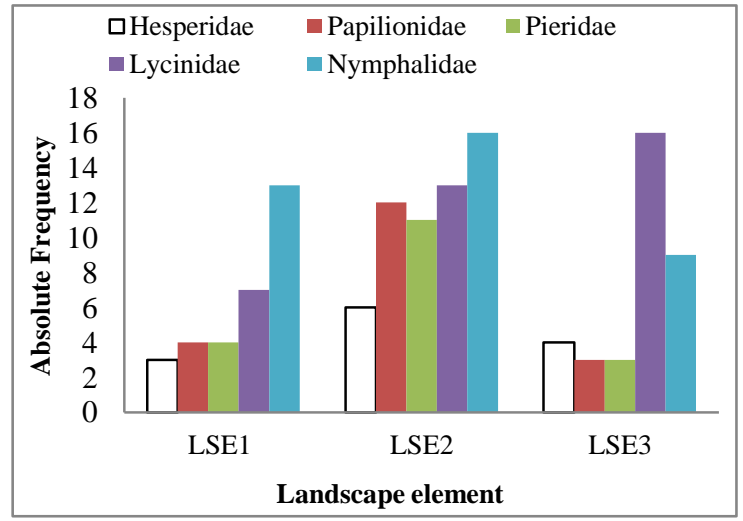

Fig. 4. Distribution of different butterfly families during pre-monsoon at different LSEs.

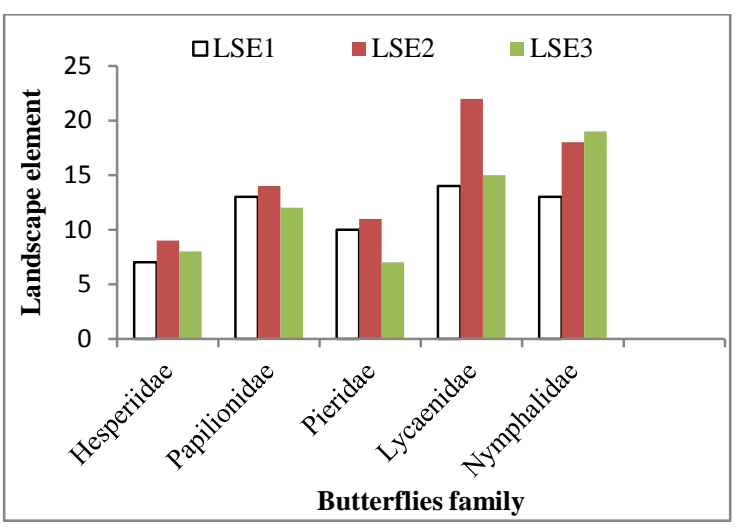

Fig. 3. Annual distribution of butterfly families at different LSEs.

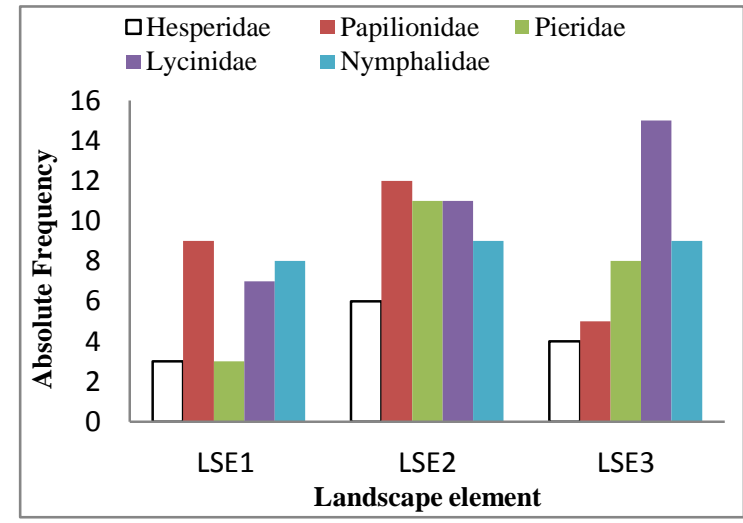

Fig. 5. Distribution of different butterfly families during post monsoon at different LSEs. 
At the transitional habitats, the habitat parameters often turn out to be less favourable due to the operation of selection forces. The selection forces are fluctuating habitat qualities, predation risk and microclimate conditions leading to the chance of its occupation by most specialized species. In contrast, the species able to thrive in multiple habitat patches with wider tolerance range in survival aspects may be treated as more generalized species. They often become able to utilize broader ecological niches; can adjust more suitably in fragmented habitats than the specialized ones through the capacity of adjustment with diverse trophic options and with less specific habitat preferences (Bashar 2015). Correlation between butterfly proboscis length and their foraging ability upon floral units are analytically depicted in order to establish the habitat preference and host selection of butterflies (Bashar 2015). A recent study has shown that 38 species of butterflies belonging to five families were recorded from invasive plant species in two study areas of West Midnapore, West Bengal, India. This study reflects the choice of butterflies towards invasive species (Paria et al. 2017). Change in land use pattern leading to changes in landscape profile may also lead to change in their diversity (Ghosh and Saha 2016).

The natural forests are recently being threatened with an alarming rate of habitat alteration, fragmentation and isolation imposed by anthropogenic overexploitation of forest resources. As a consequence climatic variation and alteration in biotope management leaded to the decline in overall biodiversity asset. In KWS, however, an overall healthy eco-potential status for butterfly populations is being maintained. This is due to the favourable ecosystem attributes maintaining a suitable landscape mosaic and ideal eco-management strategy. This strategy strikes a balance in between ecotourism and biodiversity conservation. This claim can be substantiated by the occurrence proportionately less number of butterfly species at fringe areas of Simlipal Biosphere Reserve (Payra et al. 2016) and Sunabeda Wild Life Sanctuary (Palei and Rath 2014) in respect of total area-coverage. The said results reveals in contrast to KWS with more butterfly density and diversity within a small area.

\section{ACKNOWLEDGEMENT}

Authors are thankful to the Forest Division Range, Kuldiha Wildlife Sanctuary, Odisha, India for providing spaces to carry out the research. The department of Zoology, Vidyasagar University, Midnapore, West Bengal is respectfully acknowledged for allowing to use library and laboratory facilities.

\section{REFERENCES}

Aich, U., S. Chowdhury, S. Akand, S. Rahman, K. Chowdhury, Z. Sultan and M. A. Bashar. 2016. Synchronization of coincidences between the life stages of Pachliopta aristolochiae in relation to the phenology of its host plant Aristolochia indica. J. biodivers. conserv. bioresour. manag. 2(2): 65-72.

Alam, S., M. A. Bashar, U. Aich, S. Akand and S. Rahman. 2017. Colonization and strategic stratification of butterfly Pachliopta aristolochiae (Lepidoptera: Papilionidae) and its relationship with the host plant Aristolochia indica (Piperales: Aristolochiaceae). J. biodivers. conserv. bioresour. manag. 3(1): 45-54.

Bashar, M. A. 2010. Butterflies: Best 'biotic-indicators' of climatic change. The Independent. 15(339): 27.

Bashar, M. A. 2014. Butterflies of Bangladesh: A broad approach for nature lovers. Vol. 1. 1st ed. BCTF Publications. 514 pp.

Bashar, M. A. 2015. Butterflies of Bangladesh: A broad approach for nature lovers (EBBL Red-List and butterfly activities). Vol. 2. 1st ed. BCTF Publications. 177 pp. 
Ghosh S and Saha S. 2016. Seasonal diversity of butterflies with reference to habitat heterogeneity, larval host plants and nectar plants at Taki, North 24 Parganas, West Bengal, India. World Scientific News. 50: 197-238.

Haidar, I.K.A., M.M. Rahman, M.F. Ahsan and M.A. Islam. 2017. Status, abundance and habitat preference of butterflies (Insecta: Lepidoptera) in Chittagong University Campus, Chittagong, Bangladesh. J. Threatened Taxa. 9(3): 9988-10003.

Jana, D., Giri, S., Tamili, K. D. and K. S. Chakraborty. 2013. Diversity of lepidopteran insects in the coastal regions of Midnapur (East), West Bengal, India. Indian J. Biol. Sci. 19: 32-41.

Kehimkar, I. 2008. The book of Indian butterflies. Bombay Natural History Society and Oxford University Press, Mumbai, India. 496 pp.

Koh, L. P. 2007. Impacts of land use change on South-east Asian forest butterflies: a review. J. Appl. Ecol. 44: 703-713.

Kunte, K., A. Joglekar, G. Utkarsh and P. Padmanabhan. 1999. Patterns of butterfly, bird and tree diversity in the Western Ghats. Current Science. 77: 577-586.

Leps, J. and K. Spitzer. 1990. Ecological determinants of Butterfly communities (Lepidoptera, Papilionoidea) in the Tam Dao Mountains, Vietnam. Acta Entomologica Bohemoslovaca. 87: 182194.

Lodh, R. and K. B. Agarwala. 2015. Inventory of butterfly fauna (Lepidoptera: Rhopalocera) of Tripura, India, in the Indo-Myanmar biogeographical zone, with records of threatened taxa. Check List. 11(2): 1591.

Majumder, J., R. Lodh and K. B. Agarwala. 2012. Variation in butterfly diversity and unique species richness along different habitats in Trishna Wildlife Sanctuary, Tripura, northeast India. J. Species lists. Distribut. 8(3): 432-436.

Nair. A. V., P. Mitra and S. Aditya. 2014. Studies on the diversity and abundance of butterfly (Lepidoptera: Rhopalocera) fauna in and around Sarojini Naidu college campus, Kolkata, West Bengal, India. J. Entomol. Zool. Stud. 2(4): 129-134.

Palei N. C. and B. P. Rath. 2014. Butterflies Diversity of Sunabeda Wildlife Sanctuary, Odisha, India. $J$. Entomol. Zool. Stud. 2(2): 39-44.

Paria, S., M. Bhattacharyya, H. P. Mallick and K. S. Chakraborty. 2017. Positive impact of bio-invasive host plant on butterfly diversity: the other side of the coin. ENVIS Newsletter. 23(1): 5-8.

Payra, A., G. N. Das, B. Boruah, S. K. Dash, U. P. Das and J. Sethy. 2016. Butterfly Diversity in Two Selected Fringe Area of Similipal Biosphere Reserve, Odisha, India, With Notes on Some Important Sightings. J. Wildlife Res. 4(2): 17-25.

Pollard, E. 1977. A method for assessing changes in the abundance of butterflies. Biol. Conserv. 12: 115-134.

Pollard, E. and T. J. Yates. 1993. Monitoring butterflies for ecology and conservation. Chapman and Hall, London, UK. 292 pp.

Ries, L. and T. D. Sisk. 2004. Ecological responses to habitat edges: mechanisms, models and variability explained. Ann. Rev. Ecol. Evol. Systematics. 35: 491-522. 
Singh, P. A. 2010. Butterfly diversity in tropical moist deciduous sal forests of Ankua Reserve Forest, Koina Range, Saranda Division, West Singhbhum District, Jharkhand, India. JoTT Communication. 2(9): 1130-1139.

Smetacek, P. 2012. Butterflies (Lepidoptera: Papilionoidea and Hesperoidea) and other protected fauna of Jones Estate, a dying watershed in the Kumaon Himalaya, Uttarakhand, India. JoTT Communication. 4(9): 2857-2874.

Tiple, D. A. and M. A. Khurad. 2009. Butterfly Species Diversity, Habitats and Seasonal Distribution inand Around Nagpur City, Central India. World J. Zool. 4(3): 153-162.

Varshney, R. K. and P. Smetacek. 2015. A Synoptic Catalogue of the Butterflies of India. Butterfly Research Centre, Bhimtal and Indinov Publishing, New Delhi, India. 261 pp.

DOI: http://dx.doi.org/10.3329/jbcbm.v4i1.37880 